\title{
Totally Robotic Distal Gastrectomy: A Safe and Feasible Minimally Invasive Technique for Gastric Cancer Patients Who Undergo Distal Gastrectomy
}

\author{
Yong Kuang Sanlin Lei Hua Zhao Beibei Cui Kuijie Liu Hongliang Yao \\ Department of Gastrointestinal Surgery, The Second Xiangya Hospital of Central South University, Changsha, China
}

\section{Keywords}

Gastric cancer · Distal gastrectomy · Totally robotic distal gastrectomy $\cdot$ Robotic-assisted distal gastrectomy $\cdot$ Safe

\begin{abstract}
Purposes: To explore the safety and feasibility of totally robotic distal gastrectomy (TRDG) for gastric cancer patients who undergo distal gastrectomy. Methods: Consecutive patients with gastric cancer who underwent TRDG (TRDG group) and robotic-assisted distal gastrectomy (RADG) (RADG group) were systematically reviewed at the Second Xiangya Hospital of Central South University from October 2015 to August 2018. Data were collected and statistically analyzed. Results: A total of 161 consecutive patients were included in this study: 84 cases in the TRDG group and 77 in the RADG group. Clinical characteristics and pathological results were mostly similar in both groups. The TRDG group had a significantly longer anastomotic time (20.6 \pm 3.3 vs. $17.5 \pm 4.0 \mathrm{~min}, p<0.001$ ) but showed no difference in total operating time ( $167.0 \pm 18.0$ vs. $162.9 \pm 17.6 \mathrm{~min}, p=0.159)$. The postoperative hospitalization in the TRDG group was shorter than that in the RADG group $(6.7 \pm 1.2$ vs. $7.2 \pm 1.7$ days, $p=0.019$ ). Conversion rate, estimated blood loss, and
\end{abstract}

karger@karger.com www.karger.com/dsu Karger"
(C) 2020 The Author(s)

Published by S. Karger AG, Basel

Karge

Open access

This is an Open Access article licensed under the Creative Commons Attribution-NonCommercial-4.0 International License (CC BY-NC) (http://www.karger.com/Services/OpenAccessLicense), applicable to the online version of the article only. Usage and distribution for commercial purposes requires written permission. postoperative complications were similar in both groups. There were no statistical differences in the estimated 2-year disease-free survival and overall survival rate between both groups. Conclusions: Although our current results need to be verified in further studies, TRDG represents a safe and feasible approach to distal gastrectomy and embodies the theory of minimally invasive surgery.

(C) 2020 The Author(s)

Published by S. Karger AG, Basel

\section{Introduction}

Gastric cancer is the second-most common malignancy after lung cancer in China [1]; accordingly, it has high incidence worldwide $[2,3]$. Surgery is the main treatment method for gastric cancer. At present, laparoscopic-assisted surgery has replaced open surgery as the main method for patients who undergo gastrectomy, and its superiority has been proven in multiple studies [4-6]. Although intracorporeal anastomosis (IA) in totally laparoscopic distal gastrectomy (TLDG) is more difficult to perform than totally laparoscopic colectomy, several clinical studies have demonstrated the safety and feasibility of TLDG [7-9]. 
With improvement in surgical instruments and technology, robotic-assisted distal gastrectomy (RADG) has also been reported on by experienced surgeons all over the world [10-12]. However, the safety and feasibility of totally robotic distal gastrectomy (TRDG) is not fully clear. From October 2015, TRDG and RADG were performed at the Second Xiangya Hospital of Central South University. Herein, we explore the safety and feasibility of TRDG for gastric cancer patients who underwent distal gastrectomy at our medical center.

\section{Methods}

\section{Patients and Clinical Protocol}

The study conformed to the ethical standards of the World Medical Association Declaration of Helsinki and was approved by the ethics committee at our institution. From October 2015 to August 2018, consecutive patients who were all pathologically diagnosed with gastric adenocarcinoma by biopsy and underwent TRDG and RADG were systematically reviewed. However, patients with distal metastasis and pyloric obstruction and those who underwent combined multiple organ resection were excluded from this study. All included patients accepted similar preoperative examinations such as routine blood tests; serum carcinoembryonic antigen (CEA); electrocardiograms; and pectoral, abdominal, and pelvic computed tomography scans. Before operation, detailed explanations of the pros and cons of both approaches were conveyed to patients and their families; then, one approach was chosen and consent forms were signed.

All surgeons in this study performed both types of surgery, and all of them had more than 100 cases of surgical experience with gastric cancer. Two pathologists who specialized in gastric cancer examined the surgical specimens. Tumor staging was based on the criteria from the 7th edition of the American Joint Committee on Cancer (AJCC) guidelines. Postoperative pain was evaluated by the standard clinical visual analog scale (VAS) of $0-10$, with 0 representing no pain and 10 representing the worst pain imaginable. Hemoglobin $<120 \mathrm{~g} / \mathrm{L}$ for men and $<110 \mathrm{~g} / \mathrm{L}$ for women were defined as anemia. The anastomotic leakage and duodenal stump leakage were diagnosed by digestive tract radiography. General information, operative outcomes, pathological outcomes, postoperative complications, and follow-up information of all included patients were recorded in our database.

\section{Surgical Procedure}

All operations were performed under general anesthesia with endotracheal intubation. The posture and equipment arrangement of all patients were the same as those of traditional robot surgery, with high head, low feet, and supine position. We adopted these 5 points: the $3 \mathrm{~cm}$ under the umbilicus to place a $12-\mathrm{mm}$ trocar as the observation hole; the $3 \mathrm{~cm}$ under the costal margin of the left anterior axillary line is implanted with an 8-mm trocar as Robot Arm R1; the McBurney point is placed with an 8-mm trocar as Robot Arm R2; the $2 \mathrm{~cm}$ under the costal margin of the right anterior axillary line is implanted with an $8-\mathrm{mm}$ trocar as Robot Arm $\mathrm{R} 3$; the $2 \mathrm{~cm}$ under the left midclavicular line is implanted with a 12-mm trocar as an assistant hole (Fig. 1). D2 lymph node dissec-

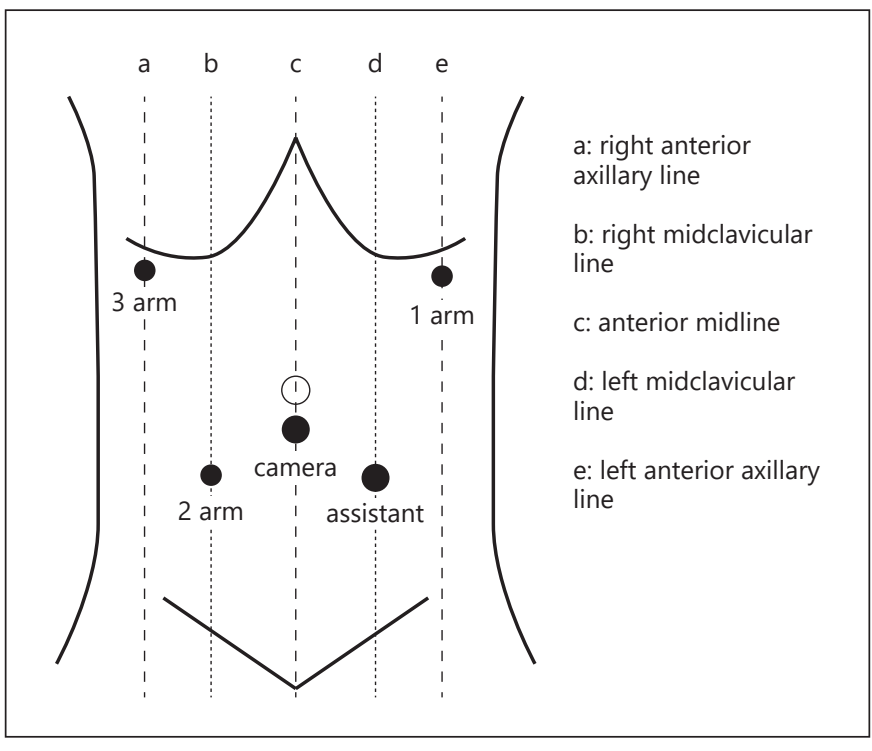

Fig. 1. Location of the trocar and incision.

tion of distal gastric cancer was performed according to preoperative staging (Fig. 2-6). Through the assistant hole, the duodenum was cut off by $2 \mathrm{~cm}$ below the pylorus and the stomach was cut off by $4 \sim 5 \mathrm{~cm}$ at the proximal end of the tumor (Fig. 7, 8). After the specimen was collected in the self-made specimen bag, the bag was tightened and placed in the right lower abdomen. After the TRDG tract reconstruction was completed, the specimen was taken out through a small incision. The specimen can also be taken out by a small incision to check whether the cutting edge is sufficient, and then TRDG tract reconstruction can be performed. During digestive tract reconstruction, make a hole at the anti-mesenteric border of the jejunum about $15 \mathrm{~cm}$ from the Treitz ligament, and another hole at the junction of the gastric stump and the greater curvature of the stomach. Side-to-side gastrojejunostomy of lesser curvature of the stomach and afferent loop was performed by an endovascular gastrointestinal anastomosis stapler (ENDO-GIA) in front of the colon (Fig. 9). The common opening was closed according to the principle of delta-shaped anastomosis by using an ENDOGIA. The completion of the whole Billroth II anastomosis is shown in Figure 10. Through the lens observation, the specimen may be taken out through a small incision.

\section{Follow-Up}

The first day after surgery was defined as the beginning of the follow-up time. After discharge, patients were advised to visit their doctors every 3 months for the first 2 years and every 6 months for the next 3 years and then have yearly visits after 5 years. The followup ended on September 20, 2018.

\section{Statistical Analysis}

Patients who required conversion were included in their intended groups because the data were analyzed based on an intention-to-treat basis. Data were analyzed by the Statistical Package for the Social Sciences (SPSS) version 24.0. Normally distributed quantitative data are presented as means \pm SD and were analyzed by Student's $t$ test; otherwise, they are expressed as median and 


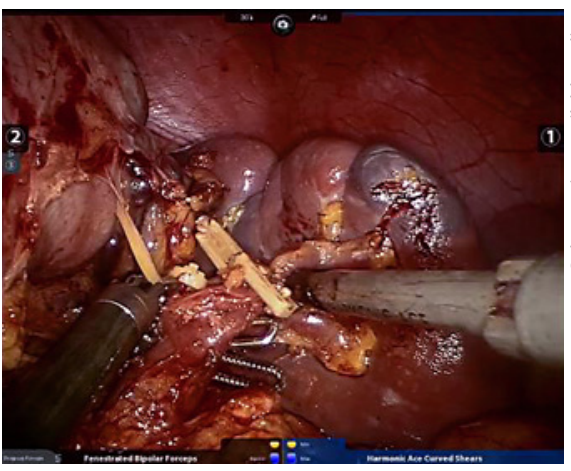

Fig. 2. Transecting gastric omental left blood vessels. Dissection of the 10, 4 sb LNs.

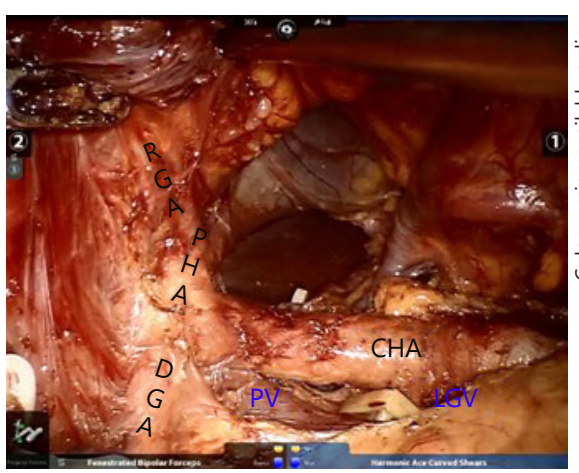

Fig. 5. Cutting the hepatogastric ligaments to expose the right gastric artery, the hepatic proper artery, the portal vein, and the common bile duct. Dissection of the 12a, 5 LNs.

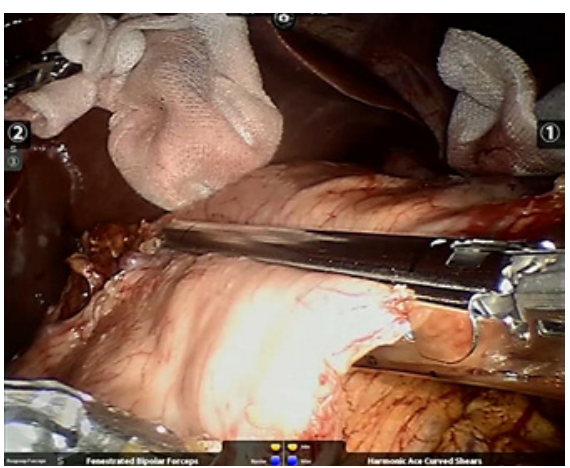

Fig. 8. The stomach was cut off by $4 \sim 5 \mathrm{~cm}$ at the proximal end of the tumor.

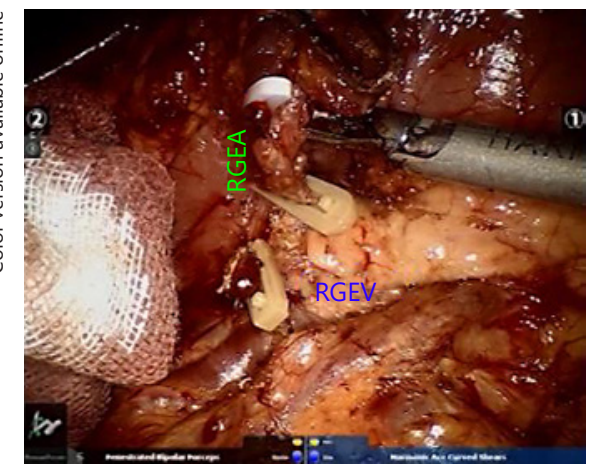

Fig. 3. Separating along the middle colon vein and its branches. Separating the adhesions of the gastric antrum and colon transverse. Dissection of the 6, 4d, $14 \mathrm{v}$ LNs.

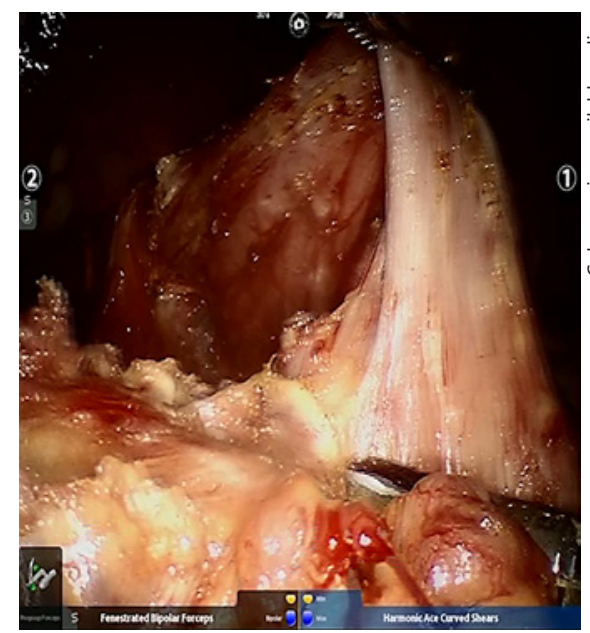

Fig. 6. Dissection of the 3, 1 LNs along the lesser curvature.

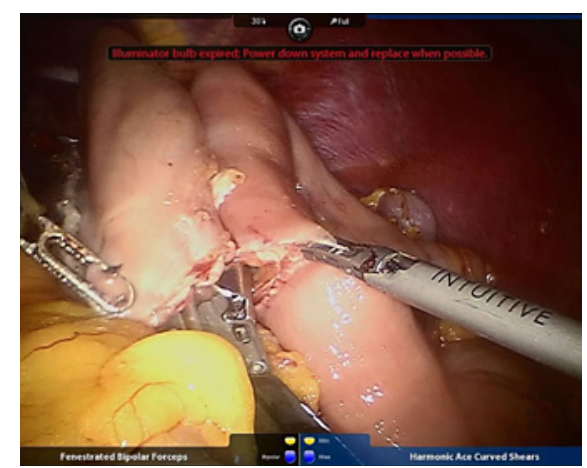

Fig. 9. Side-to-side anastomosis of the lesser curvature gastrojejunal with anterior colonic input loop.

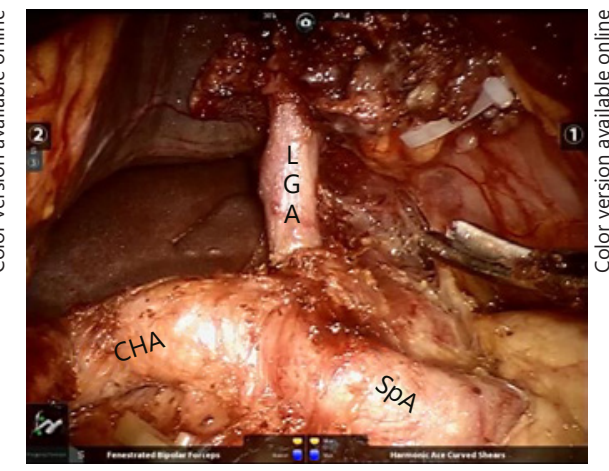

Fig. 4. Closing to the upper edge of the pancreas. Separating and exposing the splenic artery, the celiac trunk, the left gastric artery, and the common hepatic artery. Dissection of the 7, 8a, 9, 11p LNs.

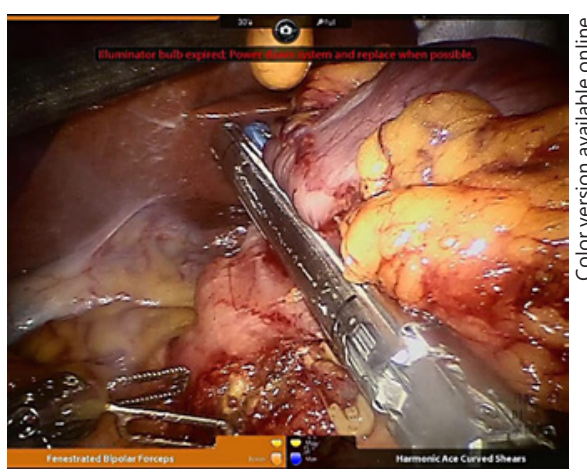

Fig. 7. Cutting off by $2 \mathrm{~cm}$ below the pylorus.

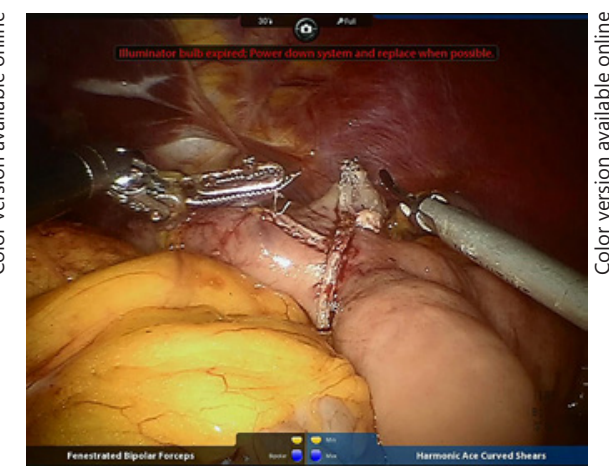

Fig. 10. The completion of the whole Billroth II anastomosis.

Kuang/Lei/Zhao/Cui/Liu/Yao 
Table 1. Clinical characteristics

\begin{tabular}{|c|c|c|c|}
\hline Parameters & $\begin{array}{l}\text { TRDG group } \\
(n=84)\end{array}$ & $\begin{array}{l}\text { RADG group } \\
(n=77)\end{array}$ & $p$ value \\
\hline Sex, $n(\%)$ & & & 0.875 \\
\hline Male & $48(57.1)$ & $45(58.4)$ & \\
\hline Female & $36(42.9)$ & $32(41.6)$ & \\
\hline Age, years & $53.3 \pm 8.9(32-69)$ & $55.5 \pm 8.4(39-72)$ & 0.105 \\
\hline $\mathrm{BMI}, \mathrm{kg} / \mathrm{m}^{2}$ & $23.9 \pm 2.2(20.4-31.6)$ & $23.6 \pm 2.0(19.8-30.2)$ & 0.375 \\
\hline ASA score, $n(\%)$ & & & 0.921 \\
\hline 1 & $38(45.2)$ & $35(45.5)$ & \\
\hline 2 & $41(48.8)$ & $36(46.8)$ & \\
\hline 3 & $5(6.0)$ & $6(7.7)$ & \\
\hline CEA level, ng/mL & & & 0.678 \\
\hline$<5, n(\%)$ & $71(84.5)$ & $63(81.8)$ & \\
\hline$\geq 5, n(\%)$ & $13(15.5)$ & $14(18.2)$ & \\
\hline Preoperative chemotherapy, $n(\%)$ & $14(16.7)$ & $15(19.5)$ & 0.685 \\
\hline Preoperative anemia, $n(\%)$ & $15(17.9)$ & $10(13.0)$ & 0.514 \\
\hline Previous abdomen surgery, $n(\%)$ & $10(11.9)$ & $10(13.0)$ & 1.000 \\
\hline
\end{tabular}

TRDG, totally robotic distal gastrectomy; RADG, robotic-assisted distal gastrectomy; ASA, American Society of Anesthesiologists; CEA, carcinoembryonic antigen.

were analyzed by the Mann-Whitney $U$ test. Categorical data are presented as a number and percentage and were analyzed by the $\chi^{2}$ test or Fisher's exact test, and ranked data were analyzed by the Mann-Whitney $U$ test. Survival analysis was calculated by the Kaplan-Meier method and analyzed using the log-rank test. A $p$ value of less than 0.05 was used as the threshold for statistical significance.

\section{Results}

\section{General Information of the TRDG Group}

A total of 84 consecutive patients ( 48 male and $36 \mathrm{fe-}$ male; mean age, $54.1 \pm 9.1$ years) were included in the TRDG group. Among them, CEA level $\geq 5 \mathrm{~g} / \mathrm{mL}$ was found in $13(15.2 \%)$ patients, $4(16.7 \%)$ patients underwent preoperative chemotherapy, 15 (17.9\%) patients had preoperative anemia, and $10(11.9 \%)$ had previous abdominal surgery (Table 1). All 84 patients underwent TRDG surgery successfully without any subsequent laparotomy. The average anastomotic time was $20.6 \pm 3.3$ $\mathrm{min}$, and time to first flatus was $2.0 \pm 1.7$ days. The postoperative hospitalization was $7.0 \pm 1.6$ days. The postoperative pain was slight (Table 2). All patients received $\mathrm{R} 0$ resection with no positive margins. Three (3.6\%) patients who underwent preoperative chemotherapy had $<15$ lymph nodes harvested. Twenty-two (26.2\%), 31 (36.9\%), and $31(36.9 \%)$ patients were classified as stage I, stage II, and stage III, respectively, based on the 7th edition of the NCCN guidelines (Table 3). The digestive tract radiography showed that anastomotic leakage and duodenal stump leakage occurred in $2(2.4 \%)$ patients and $1(1.2 \%)$ patient, respectively. Two (2.4\%) patients underwent anastomotic stenosis, and 1 patient suffered from anastomotic bleeding. One patient with anastomotic bleeding underwent unintended secondary surgery. No patients died during the perioperative period (Table 4).

\section{Comparisons between Two Groups}

Table 1 shows that both groups had similar clinical characteristics including age, sex, BMI, ASA (American Society of Anesthesiologists) score, CEA level, preoperative chemotherapy, preoperative anemia, and previous abdominal surgery. Surgical outcomes showed that the anastomotic time was longer in the TRDG group than in the RADG groups $(20.6 \pm 3.3$ vs. $17.5 \pm 4.0 \mathrm{~min}, p<0.001)$, while the overall operating time was similar between the 2 groups ( $167.0 \pm 18.0$ vs. $162.9 \pm 17.6 \mathrm{~min}, p=0.159)$. There were no significant differences in estimated blood loss $(p=0.459)$, time to first flatus ( $p=0.482)$, time to first oral intake $(p=0.695)$, postoperative hospitalization ( $p=$ 0.880 ), and postoperative pain score. The incision length for the TRDG group was significantly shorter than that for the RADG group $(6.0 \pm 1.8$ vs. $7.4 \pm 1.9, p<0.001)$ (Table 2). The pathological results were similar with respect to the number of lymph nodes harvested $(p=0.333)$ 
Table 2. Surgical results

\begin{tabular}{|c|c|c|c|}
\hline Parameters & $\begin{array}{l}\text { TRDG group } \\
(n=84)\end{array}$ & $\begin{array}{l}\text { RADG group } \\
(n=77)\end{array}$ & $p$ value \\
\hline Operating time, min (range) & $167.0 \pm 18.6(135-205)$ & $162.9 \pm 17.6(135-225)$ & 0.159 \\
\hline Anastomotic time, min (range) & $20.6 \pm 3.3(16-30)$ & $17.5 \pm 4.0(12-34)$ & $<0.001$ \\
\hline Estimated blood loss, mL (range) & $73.8 \pm 26.2(40-180)$ & $77.0 \pm 28.6(40-180)$ & 0.459 \\
\hline Open conversion, $n(\%)$ & $4(4.8)$ & $3(3.9)$ & 1.000 \\
\hline Time to first flatus, days (range) & $2.0 \pm 1.2(1.0-13)$ & $2.3 \pm 2.0(1.0-13)$ & 0.322 \\
\hline Time to first oral intake, days (range) & $1.9 \pm 1.3(1.0-13)$ & $2.0 \pm 2.1(1.1-15)$ & 0.523 \\
\hline Postoperative hospitalization, days (range) & $6.7 \pm 1.2(5-15)$ & $7.2 \pm 1.7(5-17)$ & 0.019 \\
\hline \multicolumn{4}{|l|}{ Postoperative pain score } \\
\hline First day (range) & $3.5 \pm 1.0(2-5)$ & $3.6 \pm 1.1(2-5)$ & 0.494 \\
\hline Second day (range) & $2.1 \pm 0.7(1-3)$ & $2.2 \pm 0.7(1-3)$ & 0.264 \\
\hline Third day (range) & $0.7 \pm 0.5(0-2)$ & $0.7 \pm 0.5(0-1)$ & 0.750 \\
\hline Incision length, cm (range) & $6.0 \pm 1.8(5.3-15.2)$ & $7.4 \pm 1.9(5.8-17.5)$ & $<0.001$ \\
\hline
\end{tabular}

TRDG, totally robotic distal gastrectomy; RADG, robotic-assisted distal gastrectomy.

Table 3. Pathological results

\begin{tabular}{|c|c|c|c|}
\hline Parameters & $\begin{array}{l}\text { TRDG group } \\
(n=84)\end{array}$ & $\begin{array}{l}\text { RADG group } \\
(n=77)\end{array}$ & $p$ value \\
\hline Tumor size, cm (range) & $4.5 \pm 1.0(2.8-6.9)$ & $4.5 \pm 1.0(3.1-7.0)$ & 0.803 \\
\hline Proximal resection margin, $\mathrm{cm}$ (range) & $10.0 \pm 1.6(8.5-12.0)$ & $9.9 \pm 1.6(7.6-13.5)$ & 0.713 \\
\hline Distal resection margin, $\mathrm{cm}$ (range) & $7.2 \pm 1.3(5.4-9.6)$ & $6.9 \pm 1.6(4.8-10.2)$ & 0.130 \\
\hline Lymph nodes harvested, $n$ (range) & $23.3 \pm 4.7(12-33)$ & $22.4 \pm 4.9(11-34)$ & 0.260 \\
\hline Lymph nodes harvested $<15, n(\%)$ & $3(3.6)$ & $3(3.9)$ & 1.000 \\
\hline pTNM stage, $n(\%)$ & & & 0.785 \\
\hline I & $22(26.2)$ & $18(23.4)$ & \\
\hline II & $31(36.9)$ & $30(39.0)$ & \\
\hline III & $31(36.9)$ & $29(37.6)$ & \\
\hline
\end{tabular}

TRDG, totally robotic distal gastrectomy; RADG, robotic-assisted distal gastrectomy; pTNM, pathological tumor node metastasis.

Table 4. Postoperative complications

\begin{tabular}{llll}
\hline Parameters & $\begin{array}{l}\text { TRDG group } \\
(n=84)\end{array}$ & $\begin{array}{l}\text { RADG group } \\
(n=77)\end{array}$ & $p$ value \\
\hline Anastomotic leakage, $n(\%)$ & $2(2.4)$ & $3(3.9)$ & 0.671 \\
Duodenal stump leakage, $n(\%)$ & $1(1.2)$ & $2(2.6)$ & 0.607 \\
Anastomotic stenosis, $n(\%)$ & $2(2.4)$ & $1(1.3)$ & 1.000 \\
Anastomotic bleeding, $n(\%)$ & $1(1.2)$ & $1(1.3)$ & 1.000 \\
Abdominal infection, $n(\%)$ & $2(2.4)$ & $1(1.3)$ & 1.000 \\
Gastric paralysis syndrome, $n(\%)$ & $1(1.2)$ & $2(2.6)$ & 0.607 \\
Intestinal obstruction, $n(\%)$ & $1(1.2)$ & $1(1.3)$ & 1.000 \\
Wound infection, $n(\%)$ & $2(2.1)$ & $5(6.5)$ & 0.261 \\
Reoperation, $n(\%)$ & $1(1.2)$ & $3(3.9)$ & 0.350 \\
\hline
\end{tabular}

TRDG, totally robotic distal gastrectomy; RADG, robotic-assisted distal gastrectomy. 

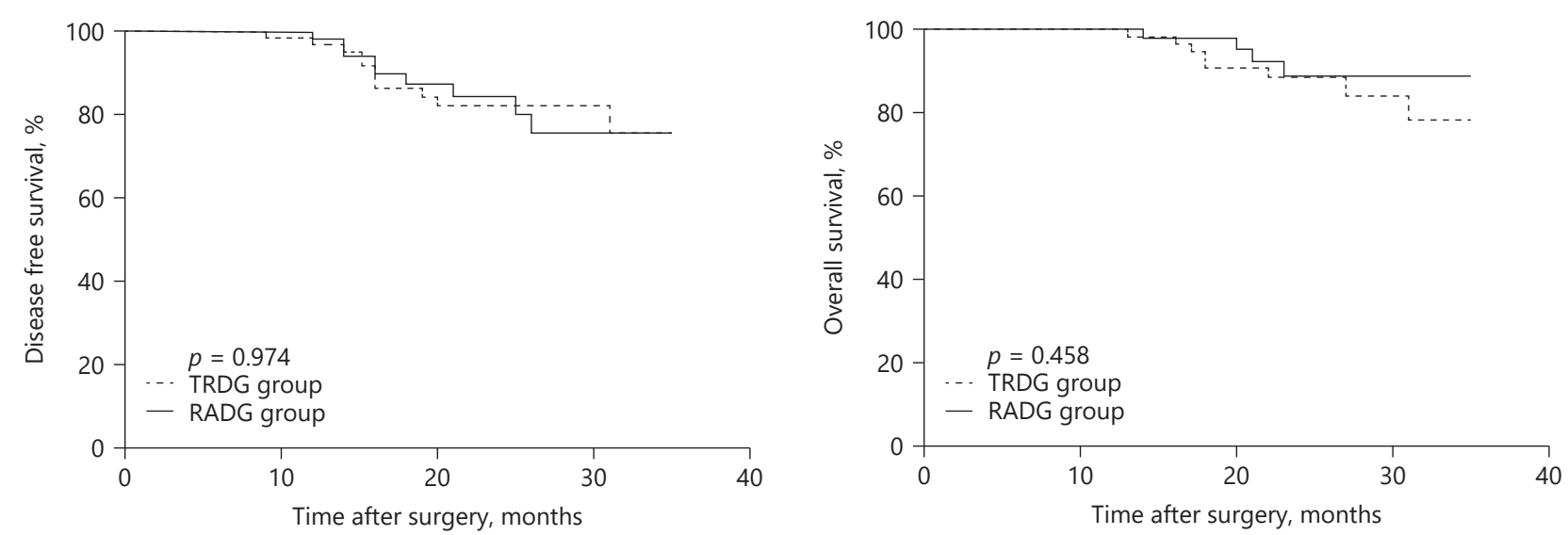

Fig. 11. Disease-free survival and overall survival of patients in the TRDG and RADG groups. TRDG, totally robotic distal gastrectomy; RADG, robotic-assisted distal gastrectomy.

and pathological tumor node metastasis $(\mathrm{pTNM})(p=$ 0.785) (Table 3). Although some patients underwent postoperative complications, including anastomotic leakage, duodenal stump leakage, anastomotic stenosis, anastomotic bleeding, abdominal infection, gastric paralysis syndrome, intestinal obstruction, and wound infection in both groups, they were not statistically significant $(p>0.05)$. Only 1 patient in the TRDG group and 3 patients in the RADG group underwent unintended secondary surgery $(p=0.350)$ (Table 4$)$.

\section{Survival Analysis}

The mean follow-up period was 20 months (range: 1-35 months) in the TRDG group and 18 months (range: $1-35$ months $)$ in the RADG group $(Z=-0.448, p=0.654)$. No patients suffered from local recurrence in the specimen extraction sites in both groups. The estimated 2-year disease-free survival rate was $82.1 \%$ in the TRDG group and $84.3 \%$ in the RADG group ( $p=0.974)$. The estimated 2 -year overall survival rate was $88.5 \%$ in the TRDG group and $88.8 \%$ in the RADG group ( $p=0.458$ ) (Fig. 11).

\section{Discussion}

The current state of gastric cancer is characterized by low rates of early diagnosis and high rates of incidence and mortality [1]. Gastric cancer is reportedly the most common malignancy of the digestive system in China [1]. Gastric antrum is the most common site of gastric cancer,

Totally Robotic Distal Gastrectomy accounting for $90 \%$ of all cases, so distal gastrectomy is the most effective treatment [13].

Laparoscopic-assisted distal gastrectomy (LADG) has the advantages of minor trauma, rapid recovery, and fewer complications than traditional open surgery, and studies have shown that for patients who underwent LADG more lymph nodes could be harvested with accompanying survival benefits [4-6]. Thus, LADG has been increasingly chosen by surgeons and patients during the past 3 decades.

Given the unceasing advances in surgical devices and gradual improvements in surgical performance, Da Vinci's surgery was tried and reported on by experienced surgeons, and its safety and feasibility were also proved [1012 ]. Suda et al. [14] showed that morbidity ( 2.3 vs. $11.4 \%$, $p=0.009)$ and hospital stay (4 [2-31] vs. 15 [8-136] days, $p=0.021)$ were significantly improved in the robotic group than in the laparoscopic group. In particular, local complication rates $(1.1$ vs. $9.8 \%, p=0.007)$ were decreased. Gao et al. [15] reported that although RADG was associated with a longer mean operating time (249.46 \pm 63.26 vs. $232.17 \pm 65.39 \mathrm{~min}, p=0.008)$ than LADG, both RADG and LADG were similar with respect to shortterm recovery and long-term oncological outcomes. Hikage et al. [16] also reported that patients in the TRDG group experienced longer operating times than the LADG group (323 vs. $285 \mathrm{~min} ; p<0.001$ ), and the incidence of all complications was similar.

However, totally robotic IA is a technically demanding procedure, making it the most important reason for 
TRDG being rarely performed. However, the IA technique has been explored by surgeons. Parisi et al. [17] adopted the "Parisi Technique" to complete reconstruction for 55 patients during totally robotic gastrectomy. The results showed that no conversions to open surgery occurred and R0 resections were obtained in all cases. In addition, the hospital stay was 5 (3-17) days, and no anastomotic leakage occurred. However, the anastomotic time was not mentioned.

In 2002, the delta-shaped anastomosis applied to TLDG was first reported by the Japanese experts Kanaya et al. [18]. They reported that all 9 patients recovered smoothly without severe complications. This new method of intracorporeal Billroth I anastomosis using only endoscopic linear staplers was proved to be simple, easy, and safe by their initial study. In 2011, he summarized that on average, only 13 min were needed to complete the intracorporeal anastomosis based on 100 cases of deltashaped anastomosis carried out by 8 surgeons; all surgeons experienced a short learning curve. In addition, he also reported that time to first oral intake was short [19]. Subsequently, an increasing number of studies confirmed the safety and feasibility of this procedure during TLDG [7-9].

In our medical center, we innovatively applied deltashaped anastomosis to TRDG in October 2015. In this study, we included 161 patients: 84 cases in the TRDG group and 77 in the RADG group. Our study showed no significant difference in total operating time, estimated blood loss, return of bowel function, and postoperative pain. For most patients in the TRDG group, the specimen was extracted via the bikini incision, which may be less conspicuous, as the incision length was significantly shortened and therefore less invasive. In addition, pathological outcomes were not compromised, with no significant difference in the number of lymph nodes harvested and the number of lymph nodes $<15$. Postoperative complications such as anastomotic leak, duodenal stump leakage, anastomotic stenosis, anastomotic bleeding, and abdominal infection occurred in both groups, but the difference was not statistically significant, and the incidence rate was similar to the reported results [14-16]. Most importantly, there were no significant differences in 2-year disease-free survival $(p=0.974)$ and overall survival rate ( $p=0.458)$ between both groups.

A limitation of our study is its retrospective nature that may have led to a bias. However, we recruited patients based on unique inclusion and exclusion criteria, and the follow-up data were complete, so we believe that our results are reliable. At present, our results represent the largest consecutive examples comparing TRDG and RADG. Randomized controlled studies in the future can verify these results.

\section{Conclusion}

Although our current results need to be verified in further studies, TRDG represents a safe and feasible approach to distal gastrectomy and embodies the theory of minimally invasive surgery.

\section{Statement of Ethics}

The study conformed to the ethical standards of the World Medical Association Declaration of Helsinki and was approved by the ethics committee at our institution. All the patients signed the operation consent and had given their written informed consent to publish the paper.

\section{Disclosure Statement}

There are no conflicts of interest to declare.

\section{Funding Sources}

This work was supported by grants from the National Natural Science Foundation of China (Nos. 81773293 and 81402536), the Natural Science Foundation of Hunan Province, PR China (2015JJ4083 and 2018JJ3758), and the Science and Technology Plan Fund in Hunan Province (2014WK2016, 2013FJ6053, and 2017WK2063).

\section{References}

1 Chen W, Zheng R, Baade PD, Zhang S, Zeng $\mathrm{H}$, Bray F, et al. Cancer statistics in China, 2015. CA Cancer J Clin. 2016;66(2):115-32.

2 Torre LA, Bray F, Siegel RL, Ferlay J, LortetTieulent J, Jemal A. Global cancer statistics, 2012. CA Cancer J Clin. 2018;65(2):87-108.
3 Sierra MS, Cueva P, Bravo LE, Forman D. Stomach cancer burden in Central and South America. Cancer Epidemiol. 2016;44(Suppl 1):S62-73.

4 Honda M, Hiki N, Kinoshita T, Yabusaki H, Abe T, Nunobe S, et al. Long-term outcomes of laparoscopic versus open surgery for clinical stage I gastric cancer: the LOC-1 study. Ann Surg. 2016; 264(2):214-22. 
5 Higgins RM, Kubasiak JC, Jacobson RA, Janssen I, Myers JA, Millikan KW, et al. Outcomes and use of laparoscopic versus open gastric resection. J Soc Laparoendosc Surg. 2015; 19(4). e2015.00095.

6 Kostakis ID, Alexandrou A, Armeni E, Damaskos C, Kouraklis G, Diamantis T, et al. Comparison between minimally invasive and open gastrectomy for gastric cancer in Europe: a systematic review and meta-analysis. Scand J Surg. 2017;106(1):3-20.

7 Seo HS, Jung YJ, Kim JH, Park CH, Lee HH. Three-port right-side approach-duet totally laparoscopic distal gastrectomy for uncut Roux-en-Y reconstruction. J Laparoendosc Adv Surg Tech A. 2018;28(9):1109-14.

8 Gong CS, Kim BS, Kim HS. Comparison of totally laparoscopic total gastrectomy using an endoscopic linear stapler with laparoscopic-assisted total gastrectomy using a circular stapler in patients with gastric cancer: a single-center experience. World J Gastroenterol. 2017;23(48):8553-61.

9 Sugiyama M, Oki E, Ogaki K, Morita M, Sakaguchi Y, Koga S, et al. Clinical outcomes of esophagojejunostomy in totally laparoscopic total gastrectomy: a multicenter study. Surg Laparosc Endosc Percutan Tech. 2017;27(4): e87-91.
10 Caruso S, Franceschini F, Patriti A, Roviello F, Annecchiarico M, Ceccarelli G, et al. Robot-assisted laparoscopic gastrectomy for gastric cancer. World J Gastrointest Endosc. 2017;9(1):1-11.

11 Amore Bonapasta S, Guerra F, Linari C, Annecchiarico M, Boffi B, Calistri M, et al. Robot-assisted gastrectomy for cancer. Chirurg. 2017;88(Suppl 1):12-8.

12 Shen W, Xi H, Wei B, Cui J, Bian S, Zhang K, et al. Robotic versus laparoscopic gastrectomy for gastric cancer: comparison of shortterm surgical outcomes. Surg Endosc. 2016; 30(2):574-80

13 Glenn JA, Turaga KK, Gamblin TC, Hohmann SF, Johnston FM. Minimally invasive gastrectomy for cancer: current utilization in US academic medical centers. Surg Endosc. 2015; 29(12):3768-75.

14 Suda K, Man-I M, Ishida Y, Kawamura Y, Satoh S, Uyama I. Potential advantages of robotic radical gastrectomy for gastric adenocarcinoma in comparison with conventional laparoscopic approach: a single institutional retrospective comparative cohort study. Surg Endosc. 2015;29(3):673-85.
15 Gao Y, Xi H, Qiao Z, Li J, Zhang K, Xie T, et al. Comparison of robotic- and laparoscopicassisted gastrectomy in advanced gastric cancer: updated short- and long-term results. Surg Endosc. 2018;33(2):528-34.

16 Hikage M, Tokunaga M, Makuuchi R, Irino T, Tanizawa Y, Bando E, et al. Comparison of surgical outcomes between robotic and laparoscopic distal gastrectomy for $\mathrm{cT} 1$ gastric cancer. World J Surg. 2018;42(6):1803-10.

17 Parisi A, Ricci F, Gemini A, Trastulli S, Cirocchi R, Palazzini G, et al. New totally intracorporeal reconstructive approach after robotic total gastrectomy: technical details and shortterm outcomes. World J Gastroenterol. 2017; 23(23):4293-302.

18 Kanaya S, Gomi T, Momoi H, Tamaki N, Isobe $\mathrm{H}$, Katayama $\mathrm{T}$, et al. Delta-shaped anastomosis in totally laparoscopic Billroth I gastrectomy: new technique of intraabdominal gastroduodenostomy. J Am Coll Surg. 2002;195(2):284-7.

19 Kanaya S, Kawamura Y, Kawada H, Iwasaki H, Gomi T, Satoh S, et al. The delta-shaped anastomosis in laparoscopic distal gastrectomy: analysis of the initial 100 consecutive procedures of intracorporeal gastroduodenostomy. Gastric Cancer. 2011;14(4):365-71. 